Kairos. Journal of Philosophy \& Science 21, 2019 Center for the Philosophy of Sciences of Lisbon University

\title{
A Importância da Biodiversidade para o Ecólogo
}

\author{
Maria Amélia Martins-Loução \\ Centre for Ecology, Evolution and Environmental \\ Changes. Faculdade de Ciências Universidade de \\ Lisboa. Campo Grande. C2. Piso 5. 1749-016 Lisboa \\ maloucao@fc.ul.pt
}

Cristina Branquinho

Centre for Ecology, Evolution and Environmental Changes. Faculdade de Ciências Universidade de Lisboa. Campo Grande. C2. Piso 5. 1749-016 Lisboa cmbranquinho@fc.ul.pt

Helena Serrano

Centre for Ecology, Evolution and Environmental Changes. Faculdade de Ciências Universidade de Lisboa. Campo Grande. C2. Piso 5. 1749-016 Lisboa hcserrano@fc.ul.pt

\begin{abstract}
Resumo Com a presente dominância do Homem sobre os ecossistemas, a sociedade enfrenta agora novos desafios do foro social, ético e ambiental. A Ecologia, como ciência transversal e holística, tem muito a contribuir para a construção do conhecimento científico, aquisição de dados e elaboração de propostas de resolução de problemas ambientais com vista a diluir o ecocentrismo e catastrofismo. A ecologia enquanto ciência pode e deve mostrar as relações entre espécies, entre comunidades e ecossistemas, entre o Homem e a natureza, minimizando noções de destruição absolutistas, e contribuindo para um diálogo científico sobre ferramentas de gestão e conservação. Este é o contributo que se pretende dar com este artigo, através de dois casos de estudo, que permitirão sugerir novas medidas de gestão e atitudes sociais perante problemas reais.
\end{abstract}

DOI 10.2478/kjps-2019-0004

Ә Open Access. (C) 2019 M.A. Martins-Loução, C. Branquinho, H. Serrano, published by Sciendo. (c))BY-NC-ND This work is licensed under the Creative Commons Attribution-NonCommercial-NoDerivatives 4.0 License. 


\section{Preâmbulo}

Biodiversidade pode ser considerado um neologismo, criado no fim da década de 80 do séc. XX por Edward O. Wilson, quando editou um livro com este nome, na sequência do Fórum do National Research Council (NRC). Este nome foi criado por simplificação do termo "biological diversity" sugerido por Thomas Lovejoy no prefácio do livro Conservation Biology'. Em 1992, na Conferência das Nações Unidas sobre o Meio Ambiente e o Desenvolvimento, realizada no Rio de Janeiro, o termo estava já interiorizado e foi adoptado para a constituição da Convenção da Biodiversidade (CBD, Convention on Biological Diversity) ${ }^{2}$. Biodiversidade é sinónimo de diversidade biológica e refere-se a qualquer nível de variabilidade existente entre seres vivos, desde a diversidade genética de uma espécie, à diversidade entre espécies e ecossistemas ${ }^{3}$. Pode ainda referir-se aos organismos vivos ou formas fósseis. Embora considerada por muitos como uma palavra sonante, toda a investigação ecológica assenta no conceito acima referido. A caracterização da biodiversidade envolve dois processos, a observação e caracterização das unidades de variação (genes, espécies ou ecossistemas) e a quantificação da variação, dentro e entre as unidades em estudo. Apesar de parecerem distintos eles são indissociáveis, já que não se pode pensar em biodiversidade apenas em número de espécies ou de ecossistemas, sem entender a sua variação e resiliência que lhes é dada pela variabilidade genética.

A diversidade de espécies presentes no Planeta Terra é o resultado de uma longa e complexa evolução, iniciada há mais de 3,5 mil miIhões de anos, como consequência de processos geológicos evolutivos e alterações climáticas dinâmicas. As espécies que chegaram até nós foram resistindo selectivamente às sucessivas alterações, diferenciando nichos que suportam serviços dos quais o Homem depende. Em pleno séc. XXI, as alterações que o Homem impôs ao ecossistema Terra são já

1 M. E. Soulé and B. A. Wilcox, Conservation Biology: An Evolutionary-Ecological Perspective (Sunderland, Massachusetts.: Sinauer Associates., 1980).

2 Convention on Biological Diversity CBD, "Convention on Biological Diversity: Text and Annexes", (Montreal, Canada: Secretariat of the Convention on Biological Diversity, 1992).

3 Maria Amélia Martins-Loução, A Aventura Da Terra: Um Planeta Em Evolução (Lisboa: Esfera do Caos, 2011). 
bastante notórias. Apesar de o termo "crise ambiental" ser considerado muito antropomórfico, as inúmeras monitorizações, a observação in situ de espécies e o desenvolvimento de indicadores ecológicos evidenciam alterações na resiliência das espécies que, a médio-longo prazo, terão consequências ainda imprevisíveis.

Para os ecólogos importa compreender o efeito da biodiversidade nos ecossistemas, a coexistência entre espécies, o funcionamento dos ecossistemas e os limiares funcionais de resposta em diferentes condições de stress, a fim de produzir ferramentas que permitam o estabelecimento de medidas de minimização de impactos, de conservação ou até mesmo de indicadores precoces de alterações.

Devido à dominância emergente do Homem sobre os ecossistemas, a sociedade enfrenta agora novos desafios. A ciência ecológica e a academia têm muito a contribuir para a construção social de uma ética ambiental, diluir o ecocentrismo. Ou seja, levar à sociedade a noção de que o Homem é apenas mais um indivíduo, ou espécie animal, na natureza complexa onde vive. Sem respeito pelos valores naturais, a degradação ambiental vai dificultar a sobrevivência humana. A ecologia enquanto ciência pode e deve mostrar as relações entre espécies, entre comunidades e ecossistemas, entre o Homem e a natureza, informando sobre os prós- e contras de acções e problemas ambientais, muitas vezes apresentadas nos media como catástrofes inevitáveis. A ecologia deve, sobretudo, contribuir para a divulgação e clarificação do conhecimento científico, aumentando a literacia da sociedade e a base de apoio ao desenvolvimento de políticas sociais e financeiras de minimização e adaptação. Este é o contributo que se pretende dar com este artigo, através de dois casos de estudo reais.

\section{Primeiro Caso de Estudo: o Azoto (N)}

O funcionamento da natureza depende de uma cadeia complexa de interacções entre organismos e destes com o seu meio envolvente. Esta cadeia de interacções é responsável pela reciclagem dos elementos químicos, em particular do carbono $(\mathrm{C})$, azoto $(\mathrm{N})$, enxofre $(\mathrm{S})$ e fósforo (P), e afecta não só a geoquímica, mas também a química da atmosfera, com consequentes alterações a nível do clima. Foi Vladimir Vernardsky, 
geoquímico russo (1863-1945), que desenvolveu a maior parte dos trabalhos sobre o complexo funcionamento dos chamados ciclos geoquímicos, dando origem, já no séc. $X X$, ao nascimento do que ele intitulou a biogeoquímica, para que não fosse ignorada a forte influência dos seres vivos em toda esta cadeia de interacções químicas. A palavra ciclo, mantida pelo hábito, é equívoca, uma vez que a maioria dos elementos está, na sua maior parte, incluída em conjuntos complexos de ciclos e pseudo-ciclos estreitamente enredados. Vernardsky foi um dos primeiros cientistas a reconhecer que o oxigénio, azoto e dióxido de carbono presentes na atmosfera resultavam de processos biológicos e, para o facto dos organismos vivos terem a capacidade de modelar o planeta, tal como qualquer força física. O legado de Vernardsky é considerado essencial para a história da ecologia contemporânea ${ }^{4}$.

De entre os elementos químicos acima referidos, o azoto é um dos mais importantes e complexos já que, juntamente com o carbono e oxigénio, representa a base química de toda a matéria orgânica presente nos seres vivos. Por outro lado, e ao contrário dos outros dois, apesar de ser muito abundante ao nível da atmosfera, só as bactérias são capazes de usar essa forma molecular e transformá-la em formas químicas de azoto reactivo (Nr), acessíveis e disponíveis aos produtores primários (plantas e algas), ao nível do solo e das águas. O azoto é, por isso, um factor limitante à produtividade vegetal e, consequentemente, à produção de alimento. Um dos grandes desafios do séc. XX foi a criação de fertilizantes sintéticos, capazes de aumentar a produção de alimentos para consumo pela população humana. Isto foi possível através da formação de amónia e hidrogénio a partir do azoto molecular atmosférico em condições de alta temperatura e pressão. Esta formação catalítica, consequência do princípio de equilíbrio químico definido por Châtelier, foi posteriormente desenvolvida à escala industrial por Carl Bosch na empresa BASF. Não foi de estranhar a atribuição dos prémios Nobel a Fritz Haber e Carl Bosch, respectivamente em 1918 e 1931 , pelas descobertas e desenvolvimento dos processos químicos industriais que permitiram a produção de fertilizantes. As formas reactivas ( $\mathrm{Nr}$ ) são aqui definidas como todos os

4 MA. Martins-Loução, "Conceitos E Terminologias No Estudo Do Ecossistema”, Cadernos de Ecologia 3 (2001). 
compostos de azoto, à excepção da forma molecular $\left(\mathrm{N}_{2}\right)$. Isto inclui as formas oxidadas, sobretudo $\mathrm{NO}, \mathrm{NO}_{2}^{-}, \mathrm{NO}_{3}^{-}$; as formas reduzidas, $\mathrm{NH}_{4}^{+}$, $\mathrm{NH}_{3}$ e os compostos orgânicos, proteínas, aminas. O desenvolvimento de fertilizantes químicos a partir do processo Haber-Bosch revolucionou a agricultura no início do séc. $X X$, dando origem ao que se intitula de Revolução Verde. Antes, o acesso a fertilizantes era muito caro porque estava dependente das reservas naturais de azoto reactivo, particularmente guano e sal amoniacal explorados, respectivamente, no Peru e Chile. A grande maioria dos agricultores usava o conhecimento empírico da adubação natural procurando um contínuo enriquecimento do solo. Com a inovação Haber-Bosch obtiveram-se fertilizantes mais baratos e com menor dispêndio de energia, permitindo uma produção de alimentos capaz de suportar o aumento populacional (Fig. 1). A exploração dos combustíveis fósseis aumentou com a revolução industrial e, consequentemente, a produção de fertilizantes foi intensificada. Do ponto de vista social, estas modificações levaram à completa alteração da exploração e uso da terra: maior intensificação e extensificação, maior adição de fertilizantes para aumentar a produtividade por hectare. Estas alterações introduziram um excesso de azoto reactivo no ambiente, perdido não só a nível da atmosfera, mas do solo e das águas, já que apenas $48 \%$ do azoto adicionado é efectivamente usado na produção. Ou seja, há ainda grande ineficiência que carece de investigação dedicada. Em 2005, dos $100 \mathrm{Tg}\left(10^{12} \mathrm{~g}\right)$ de fertilizantes usados, foram apenas gerados $17 \mathrm{Tg}$ de proteína para consumo humano, tanto vegetal como à base de carne $\mathrm{f}^{5} \mathrm{Em}$ 2010, o total de fertilizantes adicionados a nível global atingiu o valor de $174 \mathrm{Tg}$, com um desperdício de $100 \mathrm{Tg}$, o que denota a pouca eficiência de todo o processo de produção de alimentos ${ }^{6}$. De acordo com as exigências da FAO (Food and Agriculture Organization) para 20507, é urgente o desenvolvimento de tecnologias e sistemas inovadores e eficazes, para

5 J. W. Erisman et al., "How a Century of Ammonia Synthesis Changed the World", Nature Geoscience 1 (2008).

$6 \quad$ Xin Zhang et al., "Managing Nitrogen for Sustainable Development", Nature 528, no. 7580 (2015).

$7 \quad$ N. Alexandratos and J. Bruinsma, "World Agriculture Towards 2030/2050: The 2012 Revision.", ed. Agricultural Development Economics Division of the Economic and Social Development Department Working Paper No. 12-03. (Food and Agriculture Organization of the United Nations, 2012). 


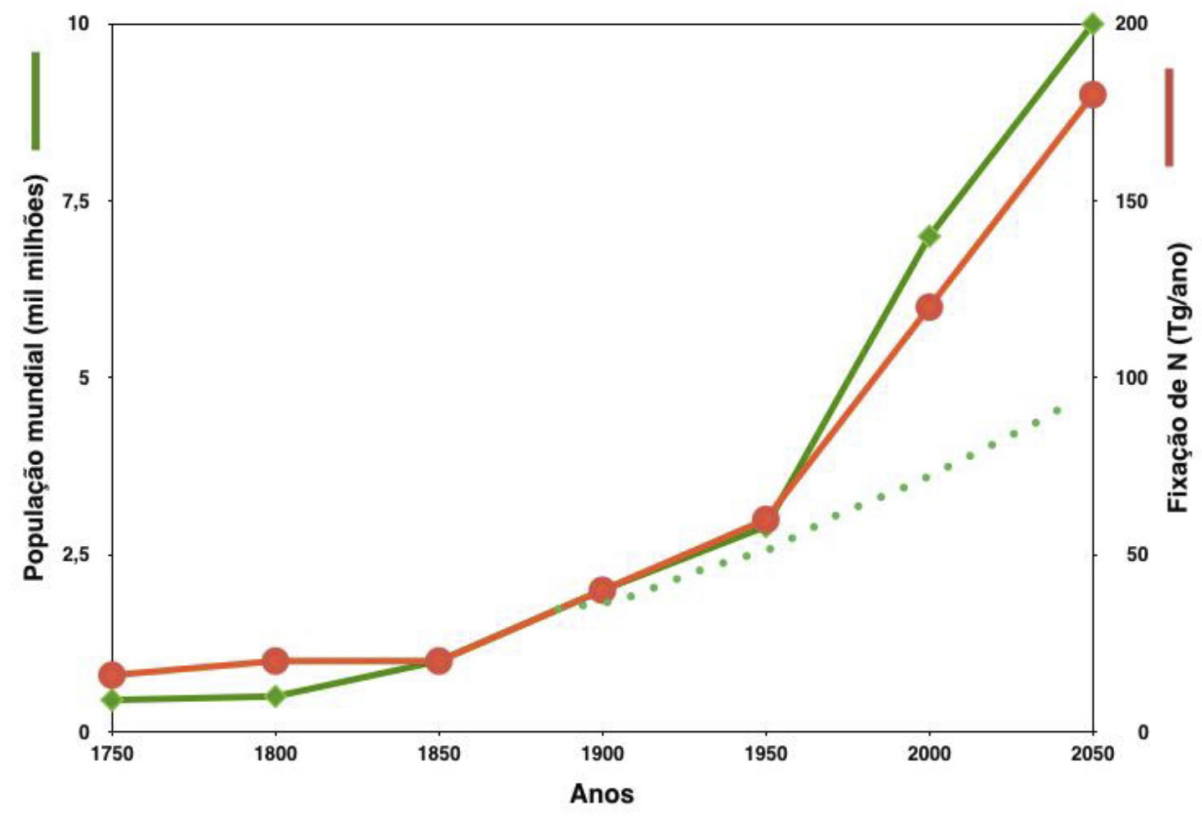

$N 2(g)+3 H 2(g) \leftarrow \rightarrow 2 N H 3(g)+$ energia

FIGURA 1 - Evolução do conhecimento científico sobre o azoto ao longo dos séculos e sua relação com o aumento da população mundial (a verde) e a fixação industrial de azoto atmosférico (a vermelho). A tracejado encontra-se a evolução da população se não tivesse sido inventado o processo de fabrico de fertilizantes. Em 1772, o $\mathrm{N}$ foi descoberto por D. Rutherford; em 1844, Liebig descreve-o como nutriente; em 1888, Beijirinck isola as primeiras bactérias fixadoras de azoto atmosférico; em 1910, Haber descobre o processo químico que permite a fixação sintética do azoto molecular em amónia; em 1913, Bosch desenvolve na BASF o processo industrial descoberto por Haber. Para comparação, a quantidade de azoto fixado naturalmente é cerca de $110 \mathrm{Tg} \mathrm{N}$. ano ${ }^{-1}$ (Adaptado de Erisman ${ }^{8}$ ).

promover uma agricultura mais inclusiva e eficiente, a fim de reduzir a pobreza rural, ajudar a eliminar a fome e a insegurança alimentar.

Por outro lado, a disponibilidade de combustíveis fósseis tornou possível a globalização e, neste momento, a produção seguida do transporte

8 Erisman et al., How a Century of Ammonia Synthesis Changed the World. Nature Geoscience, 1, 636-39 (2008). 
de bens e produtos induz a concentração de nutrientes numas regiões e a sua depleção noutras. As perdas contínuas provenientes da agricultura, indústria e transporte a nível global levaram a uma cascata de modificações na concentração dos compostos de azoto no ambiente global (Fig. 2). Nos últimos 100 anos de revolução verde ${ }^{5}$, o aumento da concentração de $\mathrm{Nr}$ no ambiente teve efeitos negativos na perda de biodiversidade $^{9}$ e na modificação da estrutura dos ecossistemas ${ }^{10,11}$, eutrofização de águas e solos, poluição de águas, acidificação, emissão de gases ${ }^{12}$ com efeito de estufa, destruição da camada de ozono, bem como a riscos de saúde pública causados por exposição a óxidos de azoto (NOx - óxido nitroso, óxido nítrico, nitrato, nitrito) e ozono. De forma a prevenir, ou remediar, os efeitos que o excesso de $\mathrm{Nr}$ tem no ecossistema foram definidas as cargas (níveis de deposição) e as concentrações máximas que um sistema pode suportar. Embora estes valores estivessem estudados para o norte da Europa, só recentemente (em 2011) foram determinados para os ecossistemas mediterrânicos. Isto foi possível usando comunidades de líquenes epifíticos, tendo por base a sua tolerância ao azoto. De posse deste conhecimento, estabeleceu-se uma classificação de grupos funcionais que foi, posteriormente, usada como ferramenta para a determinação da carga e concentrações máximas do sistema ${ }^{13}$. A calibração destes valores e sua modelação permitiram mostrar que as concentrações de $\mathrm{N}$ terão de ficar abaixo dos $1,9 \mu \mathrm{g} \cdot \mathrm{m}^{-3}$, e a carga máxima abaixo dos $26 \mathrm{~kg} \mathrm{~N} \cdot \mathrm{ha}^{-1}$. ano-1. Este resultado científico possibilita desenvolver políticas ambientais necessárias à protecção de matas e

$9 \quad$ P. Pinho et al., "Causes of Change in Nitrophytic and Oligotrophic Lichen Species in a Mediterranean Climate: Impact of Land Cover and Atmospheric Pollutants", Environmental Pollution 154, no. 3 (2008).

10 T. Dias et al., "The Strength of the Biotic Compartment in Retaining Nitrogen Additions Prevents Nitrogen Losses from a Mediterranean Maquis", Biogeosciences 9, no. 1 (2012).

11 T. Dias et al., "Linking N-Driven Biodiversity Changes with Soil N Availability in a Mediterranean Ecosystem.", Plant and Soil 341 (2011).

12 P. Pinho et al., "Assessment of Critical Levels of Atmospheric Ammonia for Lichen Diversity in Cork-Oak Woodland, Portugal", Atmospheric Ammonia: Detecting Emission Changes and Environmental Impacts (2009).

13 P. Pinho et al., "Critical Loads of Nitrogen Deposition and Critical Levels of Atmospheric Ammonia for Semi-Natural Mediterranean Evergreen Woodlands", Biogeosciences 9, no. 3 (2012). 


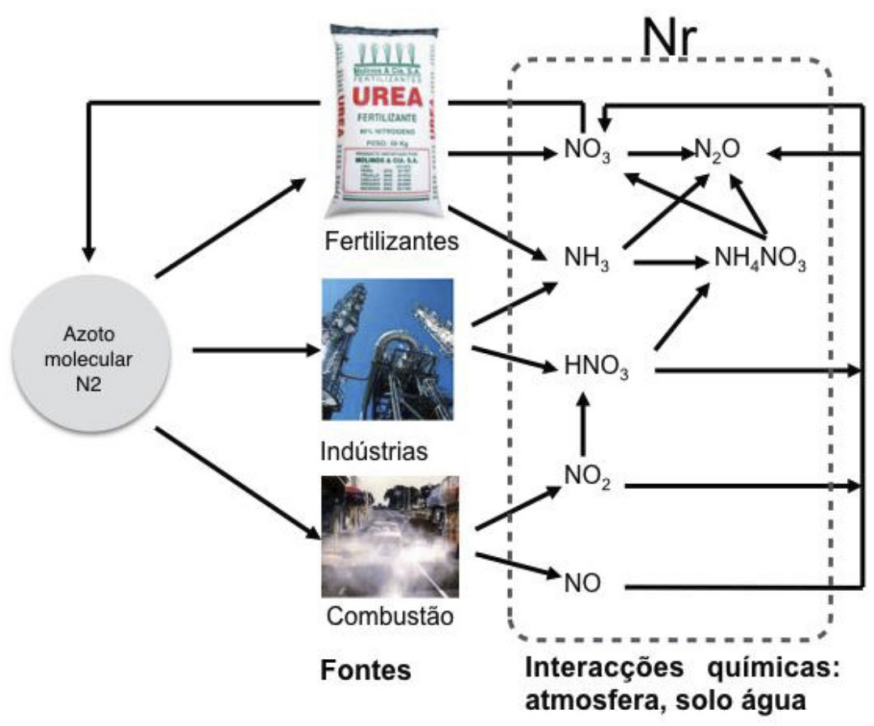

Balanço de gases: alteração climática

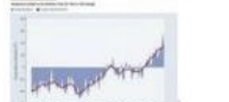

Qualidade: ar, solo, áqua

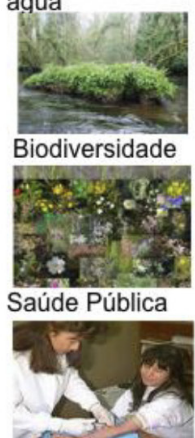

Efeitos

FIGURA 2 - Cascata de efeitos do $\mathrm{N}$ no ambiente. A molécula de azoto molecular não reactiva $\left(\mathrm{N}_{2}\right)$ passa a reactiva $(\mathrm{Nr})$ através de várias fontes perfeitamente identificadas: produção de fertilizantes, combustões várias na indústria e transportes. Estas fontes produzem diferentes formas de azoto reactivo que se perdem no ambiente e sofrem inúmeras interacções químicas ao nível da biosfera, atmosfera, água ou solo, onde produzem variados efeitos. Ao nível da atmosfera, as formas reactivas afectam o balanço de gases com efeito de estufa, incluindo o dióxido de carbono $\left(\mathrm{CO}_{2}\right)$, óxido de azoto $\left(\mathrm{N}_{2} \mathrm{O}\right)$ e metano $\left(\mathrm{CH}_{4}\right)$. Ao longo de um período de 100 anos, o $\mathrm{N}_{2} \mathrm{O}$ tem um aquecimento potencial 296 vezes maior do que igual massa de $\mathrm{CO}_{2}$, para além de contribuir para a destruição da camada de ozono. Tem por isso consequências na alteração climática. As formas reactivas têm ainda efeito directo e indirecto na Saúde Pública. A contaminação de nitratos e eutrofização de massas de água afecta a qualidade da água e a segurança alimentar. A presença de partículas de aerossol e de $\mathrm{NO}_{2}$ ao nível da atmosfera, produz efeitos tóxicos e alérgicos directos. A saturação de azoto ao nível dos solos provoca acidificação, diminuição da microflora e da qualidade do solo, com consequente limitação da produtividade. A eutrofização de águas e acidificação de solos induzem menor resiliência às espécies e alteram a estrutura dos ecossistemas. Os efeitos no ecossistema estão directamente relacionados com a perda de biodiversidade. 
matos mediterrânicos do excesso de carga de $\mathrm{N}$ e evitar, assim, a perda de diversidade e estrutura funcional destes ecossistemas ${ }^{14}$.

Em 2009, J. Rocsktröm e colaboradores ${ }^{15}$ desenvolveram um modelo conceptual para identificar e quantificar as barreiras planetárias que o Homem não deve ultrapassar sem colocar a sua segurança em risco e ajudar a clarificar o tipo de actividades humanas que mais impacte causam nos ecossistemas. Três, das nove barreiras identificadas, ultrapassaram já o limite de segurança: as mudanças climáticas, a perda de biodiversidade e a interferência humana no ciclo do azoto. A quantidade de azoto reactivo proveniente das actividades humanas é tão elevada que tem perturbado significativamente o ciclo global do azoto. Posteriormente, em $2015^{16}$ estas barreiras planetárias foram de novo equacionadas, oferecendo-se como um novo paradigma que integra o desenvolvimento contínuo da sociedade humana e a manutenção do sistema Terra num estado resiliente, usado não só para definir políticas sociais ${ }^{17}$, de preservação do ambiente ${ }^{18}$ e até mesmo económicas ${ }^{19}$. A noção de um sistema operacional seguro para a biodiversidade é vaga e pode incentivar políticas desajustadas. Por isso, cabe ao ecólogo estabelecer a conexão entre biodiversidade e estabilidade nos ecossistemas, simplificar a complexidade e divulgar informações cientificamente correctas.

No caso particular do $\mathrm{N}$, como as diferentes formas de azoto podem ser inter-convertíveis através do ambiente, um só átomo de $\mathrm{N}$ pode ter múltiplos efeitos até ser imobilizado ou eventualmente desnitrificado

14 T. Dias et al., "Species of Arbuscular Mycorrhizal Fungal Spores Can Indicate Increased Nitrogen Availability in Mediterranean-Type Ecosystems.", in Nitrogen Deposition, Critical Loads and Biodiversity, ed. Mark A. Sutton, et al. (Berlin, Dordrecht: Springer Verlag, 2014).

15 J Rockström et al., "Planetary Boundaries: Exploring the Safe Operating Space for Humanity", Ecology and Society 14, no. 2 (2009)." Ecology and Society 14, no. 2 (2009).

16 Will Steffen et al., "Planetary Boundaries: Guiding Human Development on a Changing Planet", Science 347, no. 6223 (2015).

17 Benno Keppner, "Making the Planetary Boundaries Concept Work", in Planetary Boundaries (Berlin: German Environment Agency, 2017).

18 José M. Montoya, Ian Donohue, and Stuart L. Pimm, "Planetary Boundaries for Biodiversity: Implausible Science, Pernicious Policies", Trends in Ecology \& Evolution 33, no. 2 (2018).

19 Kate Raworth, Doughnut Economics. Seven Ways to Think Like a 21 st-Century Economist (Cornerstone, 2018). 
(convertido em $\mathrm{N}_{2}$ ). Mais recentemente, Conijn e colaboradores ${ }^{20}$ aplicaram este conceito à agricultura e mostraram que o impacte da agricultura deve ser mensurado através das perdas de $\mathrm{N}$ e não da quantidade de fertilizante adicionado.

Este é um tema que merece especial atenção, tanto mais que do ponto de vista ético pode ter implicações contraditórias. Se, por um lado, o azoto é um elemento fundamental para aumentar a produção alimentar necessária a uma população crescente, por outro, é quando em excesso, um elemento perturbador com efeitos negativos para os ecossistemas.

O problema é escamoteado porque se associa a falta de alimento ao dilema da fome, omitindo-se as falhas de distribuição e os elevados custos dos desperdícios alimentares. Mais de 30\% do alimento produzido é desperdiçado com custos para o ambiente, a Saúde Pública e a equidade social. De acordo com os dados disponibilizados e sempre actualizados da $\mathrm{FAO}^{21}$, os países ricos desperdiçam tanta comida (222 milhões de toneladas) como a quantidade disponível nos países Africanos (230 milhões de toneladas). Para a Grã Bretanha calculou-se que, do ponto de vista energético, o desperdício alimentar podia ser usado para fornecer energia a cerca de 600.000 casas. Importa, porém, referir que a distribuição de alimentos também tem elevados custos ambientais, sobretudo se os locais de produção excedentária estiverem localizados muito longe dos deficitários, como acontece actualmente na esmagadora maioria dos casos. Daí a necessidade de se promoverem estratégias de retoma de tradições agrícolas em zonas rurais ou desertificadas.

Os estudos científicos sobre o efeito do azoto reactivo no ambiente têm sido tema de conferências internacionais e comunicados de imprensa ${ }^{22}$. No entanto, a influência política da comunidade científica é ainda fraca, necessitando de uma forte cooperação internacional, já que os efeitos são globais. Foram identificadas cinco áreas ameaçadas pelo excesso de $\mathrm{Nr}$ no ambiente: o balanço dos gases com efeito de estufa, a qualidade

20 J.G. Conijn et al., "Can Our Global Food System Meet Food Demand within Planetary Boundaries?", Agriculture, Ecosystems \& Environment 251, no. Supplement C (2018).

21 http://www.fao.org.

22 MA. Sutton and H. van Grinsven, "Summary for Policy Makers", in The European Nitrogen Assessment, ed. MA. Sutton, et al. (Cambridge: Cambridge University Press, 2011). 
do solo, da água e do ar, e a biodiversidade e funcionamento dos ecossistemas (Fig. 2 esquema do lado direito). Estas cinco ameaças culminaram em sete planos de acção que necessitam ser geridos de forma global e que estão relacionados com a agricultura, transportes e indústria, tratamento de águas e resíduos, e padrões sociais de consumo. O conhecimento científico e os instrumentos políticos foram desenvolvidos, mas novas estratégias de comunicação e sensibilização da sociedade necessitam ser aplicadas.

Têm sido os ecólogos que, em equipas interdisciplinares, têm vindo a chamar a atenção para o excesso de azoto reactivo e suas consequências. Quando o aumento da deposição de azoto foi detectado considerou-se que poderia ser uma fonte "barata" de fertilizante, já que a grande maioria dos solos é deficitário em azoto. No entanto, a deposição em muitos dos ecossistemas naturais tem vindo a exceder largamente o que é utilizável pela vegetação. Quando isto acontece, o azoto deixa de ser limitante para as plantas de rápido crescimento, que se tornam dominantes afectando a estrutura dos ecossistemas terrestres. Nos sistemas aquáticos, o excesso de azoto origina "blooms" de algas (crescimento excessivo) levando ao aparecimento de zonas de hipoxia, com consequências negativas para a fauna. Os impactes da deposição de azoto incluem, assim, a eutrofização de ecossistemas, acidificação dos solos, contaminação dos aquíferos por nitratos, formação de partículas atmosféricas com impacte na saúde humana, alterações do balanço de radiação, e alterações climáticas. Desta forma, uma molécula de azoto reactivo, no seu percurso ao longo dos ecossistemas terrestres e aquáticos, pode contribuir para uma cascata de efeitos, cada um dos quais com potencial para afectar a biodiversidade (Fig. 2). Os maiores impactos, $75 \%$, advêm do efeito do $\mathrm{NOx}$ e $\mathrm{NH}_{3}$ na saúde humana e nos ecossistemas.

Para além da identificação e reconhecimento da gravidade dos efeitos, que o excesso de azoto no ambiente provoca, há que compreender quais as maiores fontes "poluidoras" e tentar diminuir as emissões. A agricultura é uma das maiores fontes, mas o sector agrícola é dos mais sensíveis. A agricultura e a economia agrícola deviam ser amplamente discutidas e seriamente equacionadas do ponto de vista científico e político, pelos fortes impactos que originam a nível do clima e da biodiversidade dos 
ecossistemas. As antigas relações altruístas entre agricultores e produção, numa lógica de agricultura de subsistência, deixaram de existir.

Actualmente, a agricultura moderna contempla a produção de plantas e animais de forma intensiva. A exploração económica destes sistemas faz-se através de uma tecnologia de ponta, com a utilização de máquinas e fertilizantes, com o objectivo de atingir altos índices de produtividade. No entanto, o aumento da produção de proteína animal tem elevados custos para o Homem, tanto do ponto de vista ambiental, como de Saúde Pública. Em termos de actividade, a produção de carne e o seu processamento representam $45 \%$ das emissões de gases com efeito de estufa. Só a pecuária é responsável pela emissão de $18 \%$ do metano, gás 20 vezes mais nocivo que o dióxido de carbono $\left(\mathrm{CO}_{2}\right)$ no efeito de estufa. A nível global, a indústria da carne é das mais protegidas e subsidiadas, e uma das que se apresenta como sustentáculo da economia, responsável pela produção de alimento, independentemente dos seus impactos ambientais. E, no entanto, são poucos os que relacionam as alterações climáticas à produção de alimento, em particular à indústria da carne. Por outro lado, os riscos de uma dieta com demasiada proteína animal para a incidência de doenças cardíacas e cancerígenas são já um dado adquirido, mas pouco divulgado e que carece de uma ampla informação, sem demagogias nem utilizações políticas. Na Sociedade actual os indivíduos estão demasiado condicionados às múltiplas informações com diferentes "feedbacks" e tempos de resposta, sem se aperceber que as consequências das suas acções podem ter impactos temporais e espaciais, mais eficazes do que as políticas públicas tão fortemente exigidas. Os padrões sociais de consumo não mais poderão ser escamoteados e terão de ser abordados de forma transversal e transdisciplinar.

Todos estes problemas aqui invocados acerca do azoto, como elemento fundamental para a produção de alimento, têm tido grande desenvolvimento e avanço científicos. Falham, porém, na forma como a mensagem é transmitida, porque gera receios e incertezas sem suporte sustentável de políticas e acções concertadas. O desafio está em abdicar de slogans pré-concebidos. É preferível apresentar soluções simples a problemas reais do dia a dia, facilitar o conhecimento e catalisar verdadeiras mudanças do ponto de vista técnico, social e político para criar uma sociedade mais sustentável e com maior equidade social. E é neste 
dilema que os cientistas se devem posicionar, participando activamente na divulgação dos seus conhecimentos de forma correcta e objectiva.

\section{Segundo Caso de Estudo: Plantago almogravensis}

Os ecossistemas fornecem serviços ao Homem, que os usa e abusa de forma indiscriminada como se a biosfera fosse inesgotável, eterna e resiliente, ou seja com capacidade de resistir a condições de stress. No fundo, houve uma mudança significativa de um planeta sem espécie humana para um outro onde o Homem ocupa e usa o capital natural como moeda de troca de bens e serviços para aumentar o produto interno bruto. Em 1997 foram lançados dois trabalhos pioneiros: o livro de Gretchen Daily ${ }^{23}$, sobre o conceito de serviços do ecossistema e o de Costanza ${ }^{24}$, sobre a valorização desses mesmos serviços. Ou seja, atribuir um valor monetário às características ecológicas, funções ou processos que directa ou indirectamente contribuem para o bem estar humano, benefícios esses que derivam do funcionamento dos ecossistemas. A grande vantagem deste conceito é o de mostrar como o Homem depende da natureza - ou seja do seu capital natural - mostrando que a negligência desta dependência tem graves consequências para o ambiente e para a própria economia.

O desafio deste século é compreender que a sustentabilidade da Sociedade depende da sustentabilidade ecológica. Isto implica o reconhecimento que o capital natural e social não são substituíveis e que as barreiras planetárias propostas por Rockström²5 em 2009 são níveis inaceitáveis de degradação ambiental que colocam em causa os mínimos sociais básicos explanados nos Objectivos do Desenvolvimento Sustentável, definidos pelas Nações Unidas ${ }^{26}$. E se o azoto foi um dos fluxos que mais riscos tem trazido em termos de barreiras já ultrapassadas, a perda

23 Gretchen Daily, Nature's Services. Societal Dependence on Natural Ecosystems (Washington: Island Press, 1997).

24 R. Costanza et al., "The Value of the World's Ecosystem Services and Natural Capital", Nature 387 (1997).

25 Rockström et al., Planetary Boundaries: Exploring the Safe Operating Space for Humanity. Ecology and Society 14(2), 32-63. (2009)

26 http://www.undp.org/content/undp/en/home/sustainable-development-goals. html. 
de biodiversidade, ao ritmo a que se encontra, está a afectar a capacidade de resiliência do planeta. Também Sukhdev, em 2010, enquanto líder da Agenda Global sobre Ecossistemas e Biodiversidade, no âmbito do Fórum Mundial Económico, chamou a atenção para as consequências económico-financeiras da degradação da biodiversidade e dos ecossistemas. É nessa altura que é criado o TEEB (The Economics of Ecossystems and Biodiversity) como iniciativa global, com o objectivo de desenvolver acções que permitam manter o capital natural na agenda política e nos fóruns da economia mundial ${ }^{27}$. Tanto ao nível do TEEB como do conceito de Serviços do Ecossistema, a conservação da biodiversidade e dos ecossistemas surge como prioridade, transversal que requer um trabalho sério e objectivo por parte dos cientistas.

No entanto, o conceito de serviços do ecossistema, independentemente da sua utilidade como conceito para uma efectiva e cuidada gestão dos ecossistemas, ao promover diálogos interdisciplinares entre investigadores, nomeadamente conservacionistas, agricultores, economistas e políticos, tem suscitado muitas dúvidas do ponto de vista ético. Isto porque os investigadores ligados à conservação da natureza consideram que a conservação e a atitude de respeito para com a natureza deve ser intrínseca e não suscitada pelo valor que o ecossistema confere ${ }^{28}$. Também o TEEB, que tem como grande objectivo o desenvolvimento de uma taxa monetária sobre os serviços de ecossistema, de acordo com as regras da economia de mercado, tem vindo a levantar problemas éticos sobre a apropriação dos processos do ecossistema como produtos ou bens e não como capital natural.

Independentemente destas considerações éticas, e ao longo dos últimos $20 \operatorname{anos}^{29}$, o conceito de serviços do ecossistema veio permitir mostrar a importância da biodiversidade na sustentabilidade do ambiente. Este devia ser um tema de debate alargado entre cientistas e, simultaneamente, um conceito cujo valor, científico e prático, devia ser divulgado entre diferentes grupos sociais.

27 http://www.teebweb.org/about/the-initiative/.

28 K. Jax et al., "Ecosystem Services and Ethics", Ecological Economics 93 (2013).

29 Robert Costanza et al., "Twenty Years of Ecosystem Services: How Far Have We Come and How Far Do We Still Need to Go?", Ecosystem Services 28, no. Part A (2017). 
Porque é que a conservação da biodiversidade é assim tão importante e porque é que do ponto de vista ético os humanos devem questionar as acções que levam a uma perda de diversidade? Num ambiente de permanente mudança como actualmente se vive, com um aumento crescente de população e com recursos limitados, há uma necessidade premente de conservar os recursos genéticos para manter a produção alimentar e segurança ambiental. Ou seja, a necessidade de conservar tem um cerne antropocêntrico, embora a ecologia, como ciência holística, deva procurar desenvolver a responsabilidade moral do Homem pela exploração excessiva e descontrolada dos ecossistemas.

Quando há cerca de 10.000 anos o Homem passou de colector a agricultor, aprendeu a cultivar cerca de 10.000 espécies diferentes que lhe forneciam o alimento, os fármacos e o abrigo. Através de uma cuidada selecção, mantida e melhorada ao longo do tempo, o número de espécies em uso foi diminuindo, mas especialmente, foi sendo reduzida a sua diversidade genética. Actualmente, 30.000 espécies poderiam ser comestíveis, mas apenas 150 são comercialmente importantes. Cerca de $60 \%$ das calorias derivadas de plantas são retiradas de quatro cereais arroz, trigo, milho e sorgo ${ }^{30}$. A taxa de extinção de espécies, de perda de diversidade genética e a degradação de habitats tem sido de tal modo elevada que, em Outubro de 2010, e após a revisão da primeira Convenção da Diversidade Biológica ${ }^{31}$, foram estabelecidos em Nagoya novos objectivos para o período 2011-2020 e adoptados pela União Europeia em Maio de $2011^{32}$. Ou seja, há um comprometimento global de todos os países, e da União Europeia em particular, de procurar travar a perda de biodiversidade e a degradação dos serviços dos ecossistemas até 2020, nem que para isso haja a necessidade de se desenvolverem acções de recuperação ou de restauro de ecossistemas.

Uma das grandes limitações para o desenvolvimento das estratégias de conservação é a limitação de dados disponíveis do estado de conser-

30 http://www.fao.org.

31 "Secretariat of the Convention on Biological Diversity, "The Convention on Biological Diversity Plant Conservation Report: A Review of Progress in Implementing the Global Strategy of Plant Conservation (Gspc)", (Montreal, Canada: Secretariat of the Convention on Biological Diversity, 2009).

32 http://ec.europa.eu/environment/nature/biodiversity/strategy/index_en.htm 
vação das espécies. Em Portugal uma das grandes lacunas é a falta de uma lista vermelha de espécies da flora e uma assimilação de dados taxonómicos pela "Flora Ibérica" 33 , baseados em comparações com espécies espanholas, sem terem sido sempre confirmadas do ponto de vista filogenético, ignorando as descrições taxonómicas de antigos botânicos portugueses e a ecologia das espécies. A colheita de dados e a sua confirmação sistemática é cara e os recursos humanos especializados nesta área do saber cada vez mais escassos. Actualmente, a colecção de dados por "crowdsourcing" realizada por naturalistas voluntários e amantes da natureza, associada à moda de uma "citizen science", às facilidades de posicionamento geográfico global (GPS) presentes na maioria dos telemóveis de $3^{a}$ geração, e às câmaras fotográficas cada vez mais poderosas, tem vindo a ser usada e fomentada em muitos países, em Portugal inclusive. Apesar da grande utilidade e do indiscutível valor destas iniciativas, que permitem adquirir informações, de outra forma pouco acessíveis, a falta de organização sistemática, disciplina metodológica e conhecimento científico são limitantes para uma recolha de dados credíveis do ponto de vista científico.

No seio da comunidade de conservacionistas é consensual que a meIhor estratégia de conservação seja realizada in situ, ou seja a conservação das populações em condições naturais. Isso não deve, no entanto, impedir o esforço de conservação ex situ, em bancos de germoplasma que podem complementar a manutenção in situ ${ }^{34}$. A conservação torna-se ainda mais urgente quando se trata de espécies raras e endémicas, logo com um elevado risco de extinção. No entanto, para se desenvolver um programa de conservação há que conhecer e compreender a biologia e ecologia das espécies, clarificando assim as razões que a levam a desenvolver um tão elevado estado de ameaça. Ou seja, há que entender as relações das espécies com o ambiente, abiótico e biótico, para compreender o que leva as populações a estabilizar ou a desenvolver estratégias adaptativas perante alterações ambientais. Cabe assim à investigação ecológica avaliar de forma integrada os factores condicio-

33 http://www.floraiberica.es/.

34 A. Clemente, J. Magos Brehm, and M.A. Martins-Loução, "Conservação Ex Situ Salva Espécies Ameaçadas Da Flora Portuguesa”, El/O Botanico 5 (2011). 


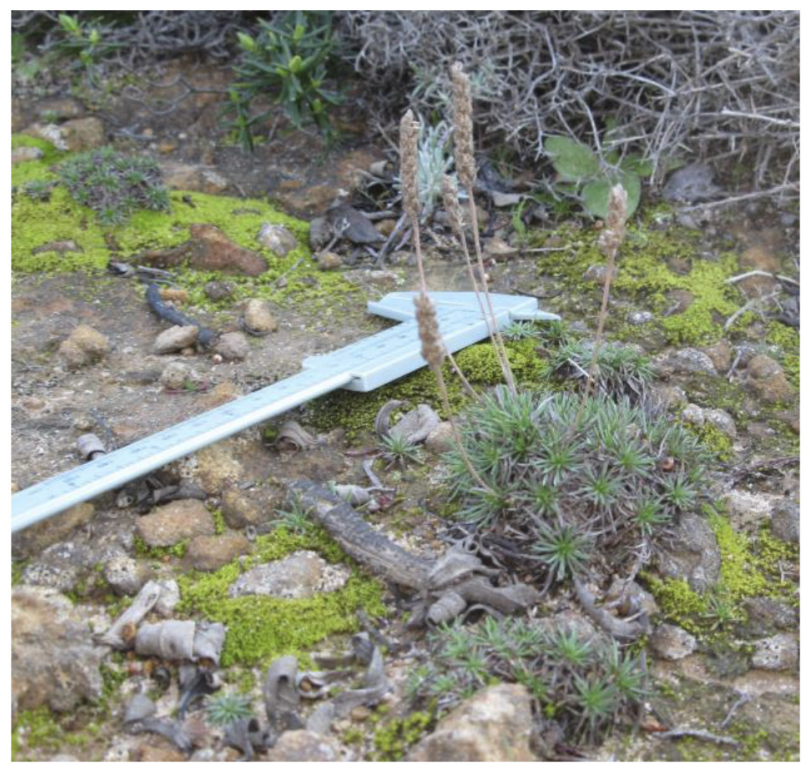

FIGURA 3 - Espécie endémica Plantago almogravensis (diabelha do Almograve). Fevereiro de 2009, H.C Serrano.

nantes duma espécie que serão determinantes para futuras estratégias de conservação ${ }^{35}$.

Plantago almogravensis Franco (Fig. 3) é uma dessas plantas incluída na lista de espécies com necessidade de medidas de conservação especial em Portugal (Directiva Habitats). A planta tem o estatuto legal de Muito Ameaçada, o que implica um elevado risco de extinção. Foram pelo menos três os factores que levaram a este estatuto: (i) estar limitada a uma única população conhecida, na costa sudoeste de Portugal; (ii) registos antigos (ca. 100 anos e mais recentes da década de 1990) sugerem a existência de outras populações, agora consideradas extintas; (iii) limitação do tamanho da actual população (ocupa $<3 \mathrm{ha}$ ). Recentemente, a sua classificação como espécie e a sua endemicidade foram postas em

35 Helena Cristina Serrano et al., "A Investigação Ecológica Como Base De Estratégias De Conservação", ibid.8 (2014). 
causa pelo lançamento da "Flora Ibérica ${ }^{36 " ~ q u e ~ a ~ j u n t o u ~ a ~ u m a ~ o u t r a ~ e s p e ́-~}$ cie de Plantago ( $P$. algarbiensis), tendo por base critérios taxonómicos, morfologicamente incompletos, não filogenéticos, e sem consideração pelo seu contexto ecológico particular.

Nos últimos 10 anos, o estudo ecológico desta espécie permitiu compreender as particularidades do seu nicho ecológico, nomeadamente no que respeita à sua relação com outras espécies e aos condicionantes ambientais do seu habitat ${ }^{37}$. $O$ reconhecimento inicial das limitações pela observação da sua morfologia e do seu habitat mostraram que Plantago almogravensis coloniza preferencialmente ilhas geoquímicas, ricas em alumínio (Al) e ferro ( $\mathrm{Fe}$ ), que formam clareiras fortemente expostas a diferentes stresses ambientais. Nestas condições, Plantago almogravensis é um acumulador de metais ${ }^{38}$, particularmente alumínio ${ }^{39}$, em tricomas glandulares de folhas e em zonas lenhosas, e desenvolve o seu nicho ecológico explorando uma área onde não tem competição com outras espécies arbustivas ${ }^{40}$. Em zonas de solos mais profundos e ricos, aparecem ocasionalmente indivíduos adultos de $P$. almogravensis, mas raramente são detectados juvenis. Estes indivíduos são "biologicamente" mais saudáveis que os das clareiras, mas não conseguem expandir o seu número porque estão rodeados de competidores fortes. Aparentemente, terão conseguido instalar-se devido à formação de aberturas temporárias no mato (ex. morte de um arbusto ou clareira de pastoreio), mas os seus descendentes tiveram de competir com as espécies arbustivas.

Pelo contrário, nas clareiras geoquímicas, a ausência de competição, permite que $P$. almogravensis se instale tolerando um solo pobre,

36 J. Pedrol, Plantago L., ed. C. Benedi, et al., Flora Ibérica (Madrid: Real Jardín Botánico CSIC, 2009).

37 Helena C. Serrano et al., "The Ecological Performance of Metallophyte Plants Thriving in Geochemical Islands Is Explained by the Inclusive Niche Hypothesis", Journal of Plant Ecology 8, no. 1 (2015).

38 H.C. Serrano et al., "How Does an Al-Hyperaccumulator Plant Respond to a Natural Field Gradient of Soil Phytoavailable Al?", Sci Total Envir 409, no. 19 (2011).

39 C. Branquinho et al., "Revisiting the Plant Hyperaccumulation Criteria to Rare Plants and Earth Abundant Elements", Environ Pollut 146, no. 2 (2007).

40 H.C. Serrano et al., The Ecological Performance of Metallophyte Plants Thriving in Geochemical Islands Is Explained by the Inclusive Niche Hypothesis. Journal of Plant Ecology, 8(1), 41-50. (2015). 
exposto e com elevado teor de alumínio. A acumulação deste metal está, aparentemente, dependente duma comunidade de microorganismos que Ihe confere alguma resiliência. Aliás, o género Plantago é conhecido por ser um dos colonizadores primários de solos pobres, pisoteados, salinos ou metalíferos. Daí a sua capacidade de colonização nestas condições tão particulares. A única população de $P$. almogravensis está restrita a estas condições particulares evidenciando elevada capacidade adaptativa. Este facto condiciona a sua capacidade evolutiva, justificando a raridade desta espécie.

Assim, as iniciativas de conservação devem ter em conta a adaptabilidade desta espécie ao habitat particular das ilhas geoquímicas onde a competição é fraca. A possibilidade de se realizarem plantações em habitats semelhantes poderá ser uma das estratégias futuras a ser implementada, já que ensaios realizados neste sentido parecem ter sido promissores ${ }^{41}$. Os esforços de conservação desta espécie poderiam ser direccionados para a reintrodução assistida e respectiva monitorização das novas populações, melhorando, paralelamente as condições ecológicas da população actual. Nomeadamente, procurar reduzir a fragmentação entre núcleos populacionais e, simultaneamente, identificar novos habitats onde a espécie possa a ser implantada.

Estas conclusões só foram possíveis depois de um estudo de investigação prolongado no tempo que permitiu compreender as características desta espécie e as suas limitações ${ }^{42}$. Claro que todas estas actividades têm custos financeiros e humanos que devem ser equacionados antes da elaboração de qualquer plano de conservação. Por essa razão, a conservação não pode nem deve ser implementada sem ter havido um estudo ecológico prévio que permita a compreensão do sistema como um todo. É importante notar que, embora seja assumida como meta europeia, a conservação e recuperação da biodiversidade para minimizar as perdas até agora registadas e manter os serviços do ecossistema, nem sempre os valores ambientais inerentes justificam o desenvolvimento de

$41 \quad$ M.J. Pinto et al., "Éxito En La Translocación De Una Planta Con Restricciones Dispersivas.", Conservación Vegetal 17 (2013).

42 H.C. Serrano, "Ecology of the Rare Endemic Plantago: Understanding the Limiting Factors Towards Its Conservation. 
estratégias de conservação a nível específico. Há conflitos e "trade-offs" entre a conservação de espécies e serviços do ecossistema que tornam as medidas de gestão e acção muito complexas e, por vezes, contraditórias, especialmente quando os custos são elevados. Mesmo entre os conservacionistas, há quem advogue o desenvolvimento de estratégias de conservação para espécies sem estatuto de ameaça e não para as raras e endémicas, já que estas estão circunscritas a um nicho muito restrito e com pouca possibilidade de aumentar a sua diversidade genética, num habitat fortemente afectado por inúmeros stresses. Estes temas de debate sobre os custos e benefícios de estratégias de conservação urgem ser discutidos para focar e direccionar planos de gestão e recuperação concertados com a mudança global que o planeta actualmente enfrenta.

\section{Conclusões}

Uma vez assumido que o Homem tem sido um agente de forte perturbação do ecossistema Terra, e que a natureza de onde o Homem retira os serviços para a sua sobrevivência é um capital natural, há todo um sentido ético ambiental que necessita ser discutido para poder responder aos objectivos do desenvolvimento sustentável debatidos e apresentados nas Nações Unidas, em Setembro de $2015^{43}$. Esta visão ética e moral é ela própria muito antropocêntrica, na medida em que a valorização que se coloca no capital natural é vista de acordo com os valores e interesses humanos. Porém, interessa salientar que, independentemente de todas as discussões do foro ético e moral, o conhecimento científico é relevante para as opções a seleccionar e para o desenvolvimento das tomadas de decisão. A capacidade de gestão é ainda fortalecida se baseada em equipas transdisciplinares que permitam trazer o conhecimento científico interdisciplinar e o discurso e a visão dos diferentes "stakeholders" (empresários, donos de terreno, agricultores, sociedade em geral) que deveriam ser envolvidos nas tomadas de decisão. Só neste tipo de fóruns a interiorização dos problemas pode ser sentida e assumida como um todo para benefício da sociedade em geral e do ambiente em particular.

43 https://news.un.org/pt/story/2015/09/1524861-novos-objetivos-de-desenvolvimento-sustentavel. 
[Nota: Os autores deste artigo não seguem o acordo ortográfico de 1990.]

\section{Referências}

Alexandratos, N., and J. Bruinsma. "World Agriculture Towards 2030/2050: The 2012 Revision.", edited by Agricultural Development Economics Division of the Economic and Social Development Department Working Paper No. 12-03.: Food and Agriculture Organization of the United Nations, 2012.

Branquinho, C., H.C. Serrano, M.J. Pinto, and M.A. Martins-Loucao. "Revisiting the Plant Hyperaccumulation Criteria to Rare Plants and Earth Abundant Elements." Environ Pollut 146, no. 2 (Mar 2007): 437-43.

$\mathrm{CBD}$, Convention on Biological Diversity. "Convention on Biological Diversity: Text and Annexes." Montreal, Canada: Secretariat of the Convention on Biological Diversity, 1992.

Clemente, A., J. Magos Brehm, and M.A. Martins-Loução. «Conservação Ex Situ Salva Espécies Ameaçadas Da Flora Portuguesa.» El/O Botanico 5 (2011): 48-49.

Conijn, J.G., P.S. Bindraban, J.J. Schröder, and R.E.E. Jongschaap. "Can Our Global Food System Meet Food Demand within Planetary Boundaries?". Agriculture, Ecosystems \& Environment 251, no. Supplement C (2018/01/01/2018): 244-56.

Costanza, R., R. d'Arge, R. de Groot, S. Farber, M. Grasso, B. Hannon, K. Limburg, et al. "The Value of the World's Ecosystem Services and Natural Capital." Nature 387 (1997): 253-60.

Costanza, Robert, Rudolf de Groot, Leon Braat, Ida Kubiszewski, Lorenzo Fioramonti, Paul Sutton, Steve Farber, and Monica Grasso. "Twenty Years of Ecosystem Services: How Far Have We Come and How Far Do We Still Need to Go?". Ecosystem Services 28, no. Part A (2017/12/01/ 2017): $1-16$.

Daily, Gretchen. Nature's Services. Societal Dependence on Natural Ecosystems. Washington: Island Press, 1997. 
Dias, T., S. Malveiro, MA. Martins-Loução, L. Shepard, and C. Cruz. "Linking N-Driven Biodiversity Changes with Soil N Availability in a Mediterranean Ecosystem.". Plant and Soil 341 (2011): 125-36.

Dias, T., M.A. Martins-Loução, L. Sheppard, and C. Cruz. "The Strength of the Biotic Compartment in Retaining Nitrogen Additions Prevents Nitrogen Losses from a Mediterranean Maquis." Biogeosciences 9, no. 1 (2012 2012): 193-201.

Dias, T., SL. Stürmer, S. Chaves, C. Fidalgo, R. Tenreiro, P. Correia, L. Carvalho, et al. "Species of Arbuscular Mycorrhizal Fungal Spores Can Indicate Increased Nitrogen Availability in Mediterranean-Type Ecosystems.". Chap. 28 In Nitrogen Deposition, Critical Loads and Biodiversity, edited by Mark A. Sutton, Kate E. Mason, Lucy J. Sheppard, Harald Sverdrup, Richard Haeuber and W. Kevin Hicks, 259-66. Berlin, Dordrecht: Springer Verlag, 2014.

Diversity, "Secretariat of the Convention on Biological. "The Convention on Biological Diversity Plant Conservation Report : A Review of Progress in Implementing the Global Strategy of Plant Conservation (Gspc)." 48. Montreal, Canada: Secretariat of the Convention on Biological Diversity, 2009.

Erisman, J.W., Mark A. Sutton, J. Galloway, Z. Klimont, and W. Winiwarter. "How a Century of Ammonia Synthesis Changed the World." Nature Geoscience 1 (2008): 636-39.

Jax, K., D.N. Barton, Kai M.A. Chan, R. de Groot, U. Doyle, U. Eser, C. Görg, et al. "Ecosystem Services and Ethics." Ecological Economics 93 (2013): 260-68.

Keppner, Benno. "Making the Planetary Boundaries Concept Work." In Planetary Boundaries, 75. Berlin: German Environment Agency, 2017.

Martins-Loução, M.A. "Conceitos E Terminologias No Estudo Do Ecossistema." Cadermos de Ecologia 3 (2001): 1-37.

Martins-Loução, Maria Amélia. A Aventura Da Terra: Um Planeta Em Evolução. Lisboa: Esfera do Caos, 2011.

Montoya, José M., Ian Donohue, and Stuart L. Pimm. "Planetary Boundaries for Biodiversity: Implausible Science, Pernicious Policies." Trends in Ecology \& Evolution 33, no. 2 (2018): 71-73. 
Pedrol, J. Plantago L. Flora Ibérica. Edited by C. Benedi, E. Rico, J. Güemes and A. Herrero Madrid: Real Jardín Botánico CSIC, 2009.

Pinho, P., S. Augusto, M.A. Martins-Loução, M.J. Pereira, A. Soares, C. Máguas, and C. Branquinho. "Causes of Change in Nitrophytic and Oligotrophic Lichen Species in a Mediterranean Climate: Impact of Land Cover and Atmospheric Pollutants." Environmental Pollution 154, no. 3 (2008 2008): 380-89.

Pinho, P., C. Branquinho, C. Cruz, Y.S. Tang, T. Dias, A.P. Rosa, C. Máguas, M.A. Martins-Loução, and M.A. Sutton. "Assessment of Critical Levels of Atmospheric Ammonia for Lichen Diversity in Cork-Oak Woodland, Portugal." Atmospheric Ammonia: Detecting Emission Changes and Environmental Impacts (2009 2009): 109-19.

Pinho, P., M.R. Theobald, T. Dias, Y.S. Tang, C. Cruz, M.A. Martins-Loução, C. Maguas, M. Sutton, and C. Branquinho. "Critical Loads of Nitrogen Deposition and Critical Levels of Atmospheric Ammonia for Semi-Natural Mediterranean Evergreen Woodlands." Biogeosciences 9, no. 3 (2012): 1205-15.

Pinto, M.J., H.C. Serrano, C. Branquinho, and M.A. Martins-Loução. "Éxito En La Translocación De Una Planta Con Restricciones Dispersivas.". Conservación Vegetal 17 (2013): 8-9.

Raworth, Kate. Doughnut Economics. Seven Ways to Think Like a 21 st-Century Economist. Cornerstone, 2018.

Rockström, J, W. Steffen, K. Noone, Å. Persson, F. Stuart III Chapin, E. Lambin, T.M. Lenton, et al. "Planetary Boundaries: Exploring the Safe Operating Space for Humanity." Ecology and Society 14, no. 2 (2009): 32-63.

Serrano, H.C. "Ecology of the Rare Endemic Plantago: Understanding the Limiting Factors Towards Its Conservation." Tese de Doutoramento em Biologia especialidade de Ecologia., Faculdade de Ciências da Lisboa, Portugal., 2015.

Serrano, H.C., M.J. Pinto, M.A. Martins-Loucao, and C. Branquinho. "How Does an Al-Hyperaccumulator Plant Respond to a Natural Field Gradient of Soil Phytoavailable Al?". Sci Total Envir 409, no. 19 (Sep 2011 ): 3749-56. 
Serrano, Helena C., Cristina Antunes, Manuel J. Pinto, Cristina Máguas, Maria Amélia Martins-Loução, and Cristina Branquinho. "The Ecological Performance of Metallophyte Plants Thriving in Geochemical Islands Is Explained by the Inclusive Niche Hypothesis." Journal of Plant Ecology 8, no. 1 (2015): 41-50.

Serrano, Helena Cristina, Manuel João Pinto, Helena Cotrim, C. Branquinho, and M.A. Martins-Loução. «A Investigação Ecológica Como Base De Estratégias De Conservação.» El/O Botanico 8 (2014): 37-39.

Soulé, M.E., and B.A. Wilcox. Conservation Biology: An EvolutionaryEcological Perspective. Sunderland, Massachusetts.: Sinauer Associates., 1980.

Steffen, Will, Katherine Richardson, Johan Rockström, Sarah E. Cornell, Ingo Fetzer, Elena M. Bennett, Reinette Biggs, et al. "Planetary Boundaries: Guiding Human Development on a Changing Planet." Science 347, no. 6223 (2015).

Sutton, MA., and H. van Grinsven. "Summary for Policy Makers." In The European Nitrogen Assessment, edited by MA. Sutton, C.M. Howard, J.W. Erisman, G. Billen, A. Bleeker, P. Grennfelt, H. van Grinsven and B. Grizzetti, XXIV-XXXIV. Cambridge: Cambridge University Press, 2011.

Zhang, Xin, Eric A. Davidson, Denise L. Mauzerall, Timothy D. Searchinger, Patrice Dumas, and Ye Shen. "Managing Nitrogen for Sustainable Development." Nature 528, no. 7580 (12/03/print 2015): 51-59. 